\title{
The construction of ladder operators and coherent states for the Wei Hua anharmonic oscillator using the supersymmetric quantum mechanics
}

\author{
Damian Mikulski • Marcin Molski • \\ Jerzy Konarski · Krzysztof Eder
}

Received: 30 May 2013 / Accepted: 16 August 2013 / Published online: 28 August 2013

(C) The Author(s) 2013. This article is published with open access at Springerlink.com

\begin{abstract}
The unknown ladder operators for the Wei Hua potential have been derived within the algebraic approach. The method is extended to include the rotating oscillator. The annihilation and creation operators have been obtained with the use of the factorization method. The coherent states for the Wei Hua anharmonic oscillator, which are eigenstates of the annihilation operator and minimize the generalized position-momentum uncertainty relation, are constructed in the framework of the supersymmetric quantum mechanics. The constructed ladder operators can be applied to compute the matrix elements in theoretical spectroscopy due to the fact that this potential is widely used to a proper description the large-amplitude vibrations of diatomic molecules.
\end{abstract}

Keywords Ladder operators · Coherent states - Wei Hua potential · Anharmonic oscillator · Supersymmetric approach · Algebraic method

\section{Introduction}

The study of the construction of coherent states and ladder operators for anharmonic oscillators, described by exactly solvable potentials has attracted much attention since the early development of quantum mechanics. A few definitions of coherent states have been proposed. The first one defines the usual coherent states as the eigenstates of

D. Mikulski $(\varangle) \cdot$ M. Molski · J. Konarski

Department of Theoretical Chemistry, Faculty of Chemistry, A. Mickiewicz University, ul. Grunwaldzka, 60-780 Poznan, Poland

e-mail: dmkwant@amu.edu.pl

D. Mikulski · K. Eder

Gen. Zamoyska and Helena Modrzejewska High School No. 2,

ul. Matejki 8/10, 60-766 Poznan, Poland 
annihilation operator for a given quantum system. The second definition of coherent states assumes the existence of a unitary displacement operator, whose action on the ground states gives the coherent states. Moreover, coherent states minimize the generalized position-momentum uncertainty relation. It should be pointed out that the latter definition relies on the form of the displacement operator, which is specific to a harmonic oscillator [1], hence in this case mainly approximated coherent states can be derived using, for example, Nieto and Simmons [2] or Kais and Levine [3-5] procedures. Also coherent states make a set of elements of a Hilbert space. All coherent states have two important properties. Firstly, they satisfy the continuity condition and resolution of identity. Secondly, they are not orthogonal to each other and are stable in time.

Note that, the ladder operators can be derived using the algebraic or supersymmetric approaches [6-9] which have been successfully applied to the Morse, Pöschl-Teller, radial harmonic and other oscillators [10-15]. Recently, Dong et al. have derived the raising and lowering operators for the Morse [16] and Pöschl-Teller potentials [17] employing some properties of the associated Laguerre and Legandre polynomials. These operators satisfy the commutation relation for the SU(2) group. The Morse potential and other ones have been studied both in terms of $\mathrm{SO}(2,1)$ and $\mathrm{SU}(2)$ Lie groups. In the $\mathrm{SO}(2,1)$ algebra the Morse Hamiltonian has been associated with the Casmir operator [18]. Also Avram and Drăgănescu [19] have obtained the ladder operators for the Morse potential, by making use of the properties of the confluent hypergeometric function in the recurrent form.

Recently, the coherent states have become a very useful tool in many fields of theoretical and experimental physics. These states have been constructed for various anharmonic oscillators, particularly for the Morse potential [4,5,16,20-23]. Perelomov [24] has been the first to discover the coherent states for quantum systems with the Lie group symmetry. Dong [25] has obtained coherent states for the Morse potential based on the SU(2) Lie algebra. Nieto and Simmons [26] presented a new method for the construction of the coherent states for arbitrary oscillators based on the classical equation of motion and applied it to the Morse oscillator. Also Benedict and Molnar [23] have presented an algebraic method employing the supersymmetric ladder operators and shape invariance method to the construction of the coherent states for the one-dimensional Morse oscillator. Moreover, they have determined the unitary displacement operator, which generates coherent states from the ground states and have constructed a new basis for the quantum systems of the Morse potential with the help of the shape invariant Lie algebra spanned by the supersymmetric ladder operators.

Gazeau and Klauder [27,28] initiated some years ago a study of coherent states for arbitrary quantum system with the discrete energy spectrum. These states are defined as eigenstates of the lowering operator of the system under consideration and are generalizations of the Barut and Girardello coherent states [29]. Crawford [30] has extended this formalism to include nondegenerated energy states. This approach has been used for the hydrogen atom. The application of the Gazau-Klauder coherent states has been developed by Antoine et al. for the Pöschl-Teller potential [31]. Also Roy and Roy [32] have constructed and examined the properties of these states for the Morse potential. Recently, Molski [33] has developed a new procedure for the 
construction of minimum uncertainty coherent states of anharmonic oscillators. This method has been adopted to the Wei Hua potential, Morse potential and Kratzer-Fues oscillator.

Molecular vibrational-rotational spectroscopy is usually known as important part of molecular physics and quantum chemistry. In real molecules the vibrations are strictly anharmonic, so if for these potentials the Schrödinger equation can be exactly solved, they may provide more suitable models for more reliable description of vibrations in diatomic molecules. One of the most important potentials in the quantum chemistry is the Wei Hua anharmonic oscillator which takes twell known form [34]

$$
V(r)=D_{e}\left(\frac{1-\exp \left(-b\left(r-r_{e}\right)\right.}{1-c \exp \left(-b\left(r-r_{e}\right)\right.}\right)^{2},
$$

in which $D_{e}$ denotes the dissociation energy of the molecular system, whereas $r_{e}$ is the equilibrium internuclear separation, $b=a(1-c)$ and $-1<c<1$. The parameter a is the range factor in the Morse potential. The parameter $\mathrm{c}$ is adjustable and minimizes the absolute deviations. The four-parameter Wei Hua oscillator is introduced for description of bond-stretching vibrations of diatomic molecules. Thus, this potential is a realistic zero order model useful for description of anharmonic vibrations in diatomic molecules. Moreover, this oscillator is a special case of the Morse potential, which is widely used in theoretical spectroscopy. This potential has the following, remarkable properties: (i) it correctly behaves for united-atom and dissociation limits, (ii) it takes into account the quasiharmonic behavior of the oscillator in the vicinity of the potential minimum, (iii) the Schrödinger equation with this potential can be exactly solved, (iv) it includes the ability of the molecules to undergo dissociation at high excitation energy, (v) absolute deviations of the Hua-Wei potential are much less than that of the Morse potential for most states. Although potential (1) provides only a crude approximation to the true molecular potential, it has many valuable advantages which make it a powerful tool for investigation of the rotation-vibrational diatomic systems. In particular, it allows the system to dissociate, which is forbidden for a harmonic oscillator and the rotation-vibrational Schrödinger equation can be exactly solved.

The first objective of the present work is to construct the ladder operators and minimum-uncertainty coherent states for the Wei Hua oscillator and generalize the approach to include the rotating oscillator. In our study we apply the Dong et al. [17] method, whereas in comparison with the study by Molski [33] in this work we have applied the standard quantum version of SUSY (so-called SUSYQM) to derive the coherent states. The work is organized as follows. In the second section based on the Dong et al. method [17] we derive ladder operators from the analytical wave functions of the Wei Hua oscillator. In the third section, this method is extended to include the rotating oscillator with nonzero angular momentum. In the fourth section the coherent states of the Wei Hua potential with zero angular momentum have been obtained. The matrix elements have been calculated with the use of these coherent states. 


\section{The ladder operators for the Wei Hua oscillator with zero total angular momentum}

In order to construct the ladder operators for the Wei Hua quantum oscillator we employ the algebraic method. The starting point in this approach is the vibrational Schrödinger equation with the Wei Hua potential (1)

$$
\left(-\frac{\hbar^{2}}{2 \mu} \frac{d^{2}}{d r^{2}}+V(r)-E_{v}\right) \Psi(r)_{v}=0 .
$$

By change of coordinate as $\mathrm{x}=c \exp \left[-b\left(r-r_{e}\right)\right]$ we rewrite Eq. (2) in the secondorder homogonous linear differential equation [34]

$$
\left[\frac{d^{2}}{d x^{2}}+\frac{1}{x} \frac{d}{d x}-\frac{t^{2}(1-Q x)^{2}}{x^{2}(1-x)^{2}}+\frac{\lambda_{v}}{x^{2}}\right] \Psi(x)_{v}=0,
$$

in which [34]

$$
t=\frac{\left(2 m D_{e}\right)^{1 / 2}}{\hbar b}, \quad \mathrm{Q}=\frac{1}{c}, \quad \frac{E_{v}}{E_{f}}=\lambda_{v}, \quad E_{f}=\frac{\hbar^{2} b^{2}}{2 \mu}
$$

The analytical solutions of this equation satisfy the following physical boundary conditions [34]

$$
\begin{array}{ll}
\Psi(x)_{v}=0 & \text { for } \mathrm{x} \in[0,1] \text { if } \mathrm{c}>0 \\
\Psi(x)_{v}=0 & \text { for } \mathrm{x} \in[-\infty, 0] \text { if } \mathrm{c}<0 .
\end{array}
$$

The analytical, normalized solution for condition (5) is the following wave function

$$
\Psi(x)_{v}=N_{v} x^{\rho_{v}}(1-x)^{1 / 2+\delta} P\left(-v, \beta_{v}, \gamma_{v} ; x\right)
$$

and for condition (6) the wave function has the form

$$
\Phi(x)_{v}=N_{v}^{\prime} x^{\rho_{v}}(1-x)^{1 / 2-\delta} P\left(-v, \beta_{v}^{\prime}, \gamma_{v} ; x\right)
$$

in which $N_{v}$ and $N_{v}^{\prime}$ denote the normalization constants, $v$ is the vibrational quantum number, whereas $P(n, a, b, x)=P_{n}^{(a, b)}(x)$ stands for the Jacoby polynomial defined by

$$
\begin{aligned}
P(n, a, b, x)= & 2^{-n} \sum_{m=0}^{n} \frac{((n+a) !)}{m !(n+a-m) !} \frac{(n+b) !}{(n-m) !(n+b-(n-m)) !} \\
& \times(x-1)^{n-m}(x+1)^{m} .
\end{aligned}
$$

In the wave functions (7) and (8) $\rho_{v}, \delta, \beta_{v}, \gamma_{v}, \beta_{v}^{\prime}$, are expressed as [34] 


$$
\begin{aligned}
\delta & =\left[\frac{1}{4}+t^{2}(Q-1)^{2}\right]^{1 / 2}, \rho_{v}=\left(t^{2}-\lambda_{v}\right)^{1 / 2}, \beta_{v}=1 / 2+\rho_{v}+\delta+\sigma, \sigma=\left(t^{2} Q^{2}-\lambda_{v}\right)^{1 / 2}, \\
\gamma_{v} & =1+2 \rho_{v}, \beta_{v}^{\prime}=1 / 2+\rho_{v}-\delta-\sigma, \\
\lambda_{v} & =\left(\delta+v+\frac{1}{2}\right)^{-2}\left\{Q t^{2}\left[v^{2}+\frac{1}{2}+v+2\left(v+\frac{1}{2}\right) \delta\right]-\frac{1}{4}\left[\left(v+\frac{1}{2}\right)^{2}+\frac{1}{4}+2\left(v+\frac{1}{2}\right) \delta\right]^{2}\right\} .
\end{aligned}
$$

The normalization constants for the wave functions (7) and (8) have the following forms [34]

$$
\begin{aligned}
& N_{v}=\left[\frac{b}{v !} \frac{\Gamma\left(2 \rho_{v}+v+1\right)}{\Gamma\left(2 \rho_{v}+1\right)} \frac{\Gamma\left(2 \delta+v+1+2 \rho_{v}\right)}{\Gamma(2 \delta+v+1) \Gamma\left(2 \rho_{v}\right)} \frac{2 \delta+2 v+1+2 \rho_{v}}{2 \delta+2 v+1}\right]^{1 / 2} \\
& N_{v}^{\prime}=\left[\frac{b \exp \left(-i \pi 2 \rho_{v}\right)}{v !} \frac{\Gamma\left(2 \rho_{v}+v+1\right)}{\Gamma\left(2 \rho_{v}+1\right)} \frac{\Gamma(2 \delta-v)}{\Gamma\left(2 \delta-v-2 \rho_{v}\right) \Gamma\left(2 \rho_{v}\right)} \frac{2 \delta-2 v-1-2 \rho_{v}}{2 \delta-2 v-1}\right]^{1 / 2} .
\end{aligned}
$$

The ladder operators can be constructed directly from the wave function (7). We are looking for the operators

$$
\stackrel{\hat{K}}{+}_{+}=\mathrm{A}_{+}(\mathrm{x}) \frac{d}{d x}+\mathrm{B}_{+}(\mathrm{x}) \text { and } \hat{K}_{-}=\mathrm{A}_{-}(\mathrm{x}) \frac{d}{d x}+\mathrm{B}_{-}(\mathrm{x})
$$

which satisfy the following relationships:

$$
\hat{K}_{+} \Psi(x)_{v}=K_{+}^{v} \Psi(x)_{v+1} \text { and } \hat{K}_{-} \Psi(x)_{v}=K_{-}^{v} \Psi(x)_{v-1}
$$

Then the raising and lowering operators can be constructed by taking advantage of the recurrent formulas of the Jacoby polynomial [35]

$$
\begin{aligned}
\frac{d}{d x} P_{n}^{(a, b)}(x)= & \frac{n[(a-b)-(2 n+a+b) x] P_{n}^{(a, b)}(x)}{(2 n+a+b)\left(1-x^{2}\right)}+\frac{2(n+a)(n+b) P_{n-1}^{(a, b)}(x)}{(2 n+a+b)\left(1-x^{2}\right)}, \\
P_{n+1}^{(a, b)}(x)= & \frac{(2 n+a+b+1)\left[(2 n+a+b)(2 n+a+b+2) x+a^{2}-b^{2}\right] P_{n}^{(a, b)}(x)}{2(n+1)(n+a+b+1)(2 n+a+b)} \\
& -\Lambda_{n} P_{n-1}^{(a, b)}(x),
\end{aligned}
$$

in which

$$
\Lambda_{\mathrm{n}}=\frac{2(n+a)(n+b)(2 n+a+b+2)}{2(n+1)(n+a+b+1)(2 n+a+b)} .
$$


Employing Eq. (15) and the result of the action of differential operators $\frac{d}{d x}$ on the wave function (7) we get

$$
\begin{gathered}
\frac{d}{d x} \Psi(x)_{v}=\frac{\rho_{v}}{x} \Psi(x)_{v}-\frac{\frac{1}{2}+\delta}{1-x} \Psi(x)_{v}+N_{v} x^{\rho_{v}}(1-x)^{1 / 2+\delta} \\
{\left[\frac{B_{v}(x)}{1-x^{2}} P_{v}^{\left(\beta_{v}, \gamma_{v}\right)}(x)+\frac{A_{v}}{1-x^{2}} P_{v-1}^{\left(\beta_{v-1}, \gamma_{v-1}\right)}(x)\right]}
\end{gathered}
$$

where $\mathrm{A}_{v}$ and $\mathrm{B}_{v}(x)$ denotes

$$
A_{v}=\frac{2\left(v+\beta_{v}\right)\left(v+\gamma_{v}\right)}{2 v+\beta_{v}+\gamma_{v}}, B_{v}(x)=\frac{v\left[\left(\beta_{v}-\gamma_{v}\right)-\left(2 v+\beta_{v}+\gamma_{v}\right) x\right]}{2 v+\beta_{v}+\gamma_{v}} .
$$

After simple calculations, we finally obtain the lowering operator in the form

$$
\begin{aligned}
\hat{K}_{-}= & x^{\rho_{v-1}-\rho_{v}}\left(1-x^{2}\right) \frac{d}{d x}-\rho_{v} x^{\rho_{v-1}-\rho_{v}-1}+(1 / 2+\delta)(1+x) x^{\rho_{v-1}-\rho_{v}} \\
& -B_{v}(x) x^{\rho_{v-1}-\rho_{v}}
\end{aligned}
$$

whereas its coefficient is given by

$$
K_{-}^{v}=A_{v} \frac{N_{v}}{N_{v-1}}
$$

The form of Eq. (20) clearly indicates that the lowering operator annihilates the ground state $\hat{K}_{-} \Psi(x)_{0}=0$.

In a similar manner using Eqs. (15) and (16), we construct the raising operator. In view of the above, the raising operator can be given in the form

$$
\begin{aligned}
\hat{K}_{+}= & \left(1-x^{2}\right) x^{\rho_{v+1}-\rho_{v}} \frac{d}{d x}-\rho_{v} x^{\rho_{v+1}-\rho_{v}-1}\left(1-x^{2}\right)+(1 / 2+\delta)(1+x) x^{\rho_{v+1}-\rho_{v}} \\
& -\left(B_{v}(x)+A_{v} \Omega_{v}(x)\right) x^{\rho_{v+1}-\rho_{v}}
\end{aligned}
$$

in which

$$
\Omega_{v}(x)=\frac{\left(2 v+\beta_{v}+\gamma_{v}+1\right)\left[\left(2 v+\beta_{v}+\gamma_{v}\right)\left(2 v+\beta_{v}+\gamma_{v}+2\right) x+\beta_{v}^{2}-\gamma_{v}^{2}\right]}{2\left(v+\beta_{v}\right)\left(v+\gamma_{v}\right)\left(2 v+\beta_{v}+\gamma_{v}+2\right)}
$$

The coefficient of the raising operator (22) is given by

$$
K_{+}^{v}=A_{v} C_{v} \frac{N_{v}}{N_{v+1}}
$$


where

$$
C_{v}=\frac{2(v+1)\left(v+\beta_{v}+\gamma_{v}+1\right)\left(2 v+\beta_{v}+\gamma_{v}\right)}{2\left(v+\beta_{v}\right)\left(v+\gamma_{v}\right)\left(2 v+\beta_{v}+\gamma_{v}+2\right)} \text {. }
$$

We now study the ladder operators, which we can construct directly from the wave function (8). To this aim, we are looking for operators in the following forms:

$$
\hat{L_{+}}=\mathrm{C}_{+}(\mathrm{x}) \frac{d}{d x}+\mathrm{D}_{+}(\mathrm{x}) \text { and } \hat{L}_{-}=\mathrm{C}_{-}(\mathrm{x}) \frac{d}{d x}+\mathrm{D}_{-}(\mathrm{x})
$$

These operators should satisfy

$$
{\hat{L_{+}}} \Phi(x)_{v}=L_{+}^{v} \Phi(x)_{v+1} \text { and } \hat{L}_{-} \Phi(x)_{v}=L_{-}^{v} \Phi(x)_{v-1} .
$$

After similar calculations, we finally obtain the following expression for the lowering, the raising operator and its coefficient

$$
\begin{aligned}
\hat{L_{-}}= & x^{\rho_{v-1}-\rho_{v}}\left(1-x^{2}\right) \frac{d}{d x}-\rho_{v} x^{\rho_{v-1}-\rho_{v}-1}\left(1-x^{2}\right)+(1 / 2-\delta)(1+x) x^{\rho_{v-1}-\rho_{v}} \\
& -B_{v}^{\prime}(x) x^{\rho_{v-1}-\rho_{v}}, \\
\hat{L_{+}}= & \left(1-x^{2}\right) x^{\rho_{v+1}-\rho_{v}} \frac{d}{d x}-\rho_{v} x^{\rho_{v+1}-1}\left(1-x^{2}\right)+(1 / 2-\delta)(1+x) x^{\rho_{v+1}-\rho_{v}} \\
& -\left(B_{v}^{\prime}(x)+A_{v}^{\prime} \Omega_{v}^{\prime}(x)\right) x^{\rho_{v+1}-\rho_{v}} \\
L_{+}^{v}= & A_{v}^{\prime} C_{v}^{\prime} \frac{N_{v}^{\prime}}{N_{v+1}^{\prime}}, L_{-}^{v}=A_{v}^{\prime} \frac{N_{v}^{\prime}}{N_{v-1}^{\prime}},
\end{aligned}
$$

in which

$$
\begin{gathered}
\Omega_{v}^{\prime}(x)=\frac{\left(2 v+\beta_{v}^{\prime}+\gamma_{v}+1\right)\left[\left(2 v+\beta_{v}^{\prime}+\gamma_{v}\right)\left(2 v+\beta_{v}^{\prime}+\gamma_{v}+2\right) x+\beta_{v}^{\prime 2}-\gamma_{v}^{2}\right]}{2\left(v+\beta_{v}^{\prime}\right)\left(v+\gamma_{v}\right)\left(2 v+\beta_{v}^{\prime}+\gamma_{v}+2\right)} \\
A_{v}^{\prime}=\frac{2\left(v+\beta_{v}^{\prime}\right)\left(v+\gamma_{v}\right)}{2 v+\beta_{v}^{\prime}+\gamma_{v}}, C_{v}^{\prime}=\frac{2(v+1)\left(v+\beta_{v}^{\prime}+\gamma_{v}+1\right)\left(2 v+\beta_{v}^{\prime}+\gamma_{v}\right)}{2\left(v+\beta_{v}^{\prime}\right)\left(v+\gamma_{v}\right)\left(2 v+\beta_{v}^{\prime}+\gamma_{v}+2\right)} \\
B_{v}^{\prime}(x)=\frac{v\left[\beta_{v}^{\prime}-\gamma_{v}-\left(2 v+\beta_{v}^{\prime}+\gamma_{v}\right) x\right]}{2 v+\beta_{v}^{\prime}+\gamma_{v}} .
\end{gathered}
$$

Additionally, one can show that the lowering operator (28) annihilates the ground state $\hat{L}_{-} \Phi(x)_{0}=0$. In the further study we will investigate the dynamical algebra associated with the considered quantum system. 


\section{The ladder operators for the rotating Wei Hua oscillator}

The starting point for derivation of the ladder operators of the rotating Wei Hua oscillator is the rotation-vibrational Schrödinger equation

$$
\left[-\frac{\hbar^{2}}{2 \mu} \frac{d^{2}}{d r^{2}}+\frac{J(J+1) \hbar^{2}}{2 \mu r^{2}}+V(r)-E_{v J}\right] \Psi(r)_{v J}=0 .
$$

The analytical, normalized solution of this equation can be written as [31]

$$
\Psi(x)_{v J}=N_{v J} x^{\rho_{v J}}(1-x)^{1 / 2+\delta_{c J}} P\left(-v, \beta_{v J}, \gamma_{v J} ; x\right),
$$

where $\mathrm{N}_{v J}$ is the normalization constant, $\mathrm{J}=0,1,2, \ldots$ is the rotational quantum number and $P(n, a, b, x)=P_{n}^{(a, b)}(x)$ stands for the Jacoby polynomial. Moreover, in the wave function (35) the parameters; $\rho_{v J}, \beta_{v J}, \gamma_{v J}, \delta_{c J}$ can be written by [34]

$$
\begin{gathered}
\rho_{v J}=\left(t^{2} \xi_{0}-\lambda_{v J}\right)^{1 / 2}, \beta_{v J}=1+v+2 \rho_{v J}+2 \delta_{c J}, \gamma_{v J}=1+2 \rho_{v J}, \\
\delta_{c J}=\operatorname{sgn}(c) \rho_{J}, \rho_{J}=\left[\frac{1}{4}+t^{2}\left(\zeta_{0}+\zeta_{1}+\zeta_{2}\right)\right]^{1 / 2}
\end{gathered}
$$

in which [33]

$$
\begin{aligned}
\zeta_{0} & =1+\frac{B_{e} J(J+1)}{D_{e}}\left[1+\frac{c^{2}+2 c-3}{b r_{e}}+\frac{3(1-c)^{2}}{b^{2} r_{e}^{2}}\right], \\
\zeta_{1} & =-Q\left[2+\frac{B_{e} J(J+1)}{D_{e}}\left[2 c+\frac{4\left(c^{2}-1\right)}{b r_{e}}+\frac{6(1-c)^{2}}{b^{2} r_{e}^{2}}\right]\right], \\
\zeta_{2} & =Q^{2}\left[1+\frac{B_{e} J(J+1)}{D_{e}}\left[c^{2}+\frac{3 c^{2}-2 c-1}{b r_{e}}+3 \frac{(1-c)^{2}}{b^{2} r_{e}^{2}}\right]\right], \lambda_{v J}=\frac{E_{v J}}{E_{f}} \\
\lambda_{v J} & =\frac{1}{2} t^{2}\left(\zeta_{0}+\zeta_{2}\right)-\frac{1}{4}\left(\delta_{c J}+v+\frac{1}{2}\right)^{2}-\frac{1}{4} t^{4}\left(\delta_{c J}+v+\frac{1}{2}\right)^{-2}\left(\zeta_{2}-\zeta_{0}\right)^{2}
\end{aligned}
$$

$B_{e}$ is the well- know equilibrium rotation constant and has the following form

$$
B_{e}=\frac{\hbar^{2}}{2 \mu r_{e}^{2}}
$$

The ladder operators for the rotating Wei Hua oscillator can be constructed directly from the wave function (35). To this aim, we are looking for the operators in the following forms:

$$
\hat{K}_{+}^{v J}=\mathrm{E}_{+}(\mathrm{x}) \frac{d}{d x}+\mathrm{F}_{+}(\mathrm{x}) \text { and } \hat{K}_{-}^{v J}=\mathrm{E}_{-}(\mathrm{x}) \frac{d}{d x}+\mathrm{F}_{-}(\mathrm{x})
$$


These operators satisfy the following properties:

$$
\hat{K}_{+}{ }^{v J} \Psi(x)_{v J}=K^{v J}+\Psi(x)_{v+1, J} \text { and } \hat{K}_{-}{ }^{v J} \Psi(x)_{v J}=K^{v J}-\Psi(x)_{v-1, J}
$$

Making use of the Eqs. (15) and (16), similarly as in the previous section, we can readily get the following expressions for the lowering operator, the raising operator and their coefficients, respectively

$$
\begin{aligned}
\hat{K}_{-}{ }^{v J}= & x^{\rho_{v-1, J}-\rho_{v J}}\left(1-x^{2}\right) \frac{d}{d x}-\rho_{v J} x^{\rho_{v-1, J}-\rho_{v J}-1}\left(1-x^{2}\right) \\
& +\left(1 / 2+\delta_{c J}\right)(1+x) x^{\rho_{v-1, J}-\rho_{v J}}-B_{v J}(x) x^{\rho_{v-1, J}-\rho_{v J}} \\
\hat{K}_{+}{ }^{v J}= & \left(1-x^{2}\right) x^{\rho_{v+1, J}-\rho_{v J}} \frac{d}{d x}-\rho_{v J} x^{\rho_{v+1, J}-\rho_{v J}-1}\left(1-x^{2}\right) \\
& +\left(1 / 2+\delta_{c J}\right)(1+x) x^{\rho_{v+1, J}-\rho_{v J}}-\left(B_{v J}(x)+A_{v J} \Omega_{v J}(x)\right) x^{\rho_{v+1, J}-\rho_{v J}} \\
K_{+}{ }^{v J}= & A_{v J} C_{v J} \frac{N_{v J}}{N_{v+1, J}}, K_{-}{ }^{v J}=A_{v J} \frac{N_{v J}}{N_{v-1, J}}
\end{aligned}
$$

in which

$$
\begin{gathered}
\Omega_{v J}(x)=\frac{\left(2 v+\beta_{v J}+\gamma_{v J}+1\right)\left[\left(2 v+\beta_{v J}+\gamma_{v J}\right)\left(2 v+\beta_{v J}+\gamma_{v J}+2\right) x+\beta_{v J}^{2}-\gamma_{v J}^{2}\right]}{2\left(v+\beta_{v J}\right)\left(v+\gamma_{v J}\right)\left(2 v+\beta_{v J}+\gamma_{v J}+2\right)}, \\
A_{v J}=\frac{2\left(v+\beta_{v J}\right)\left(v+\gamma_{v J}\right)}{2 v+\beta_{v J}+\gamma_{v J}}, C_{v J}=\frac{2(v+1)\left(v+\beta_{v J}+\gamma_{v J}+1\right)\left(2 v+\beta_{v J}+\gamma_{v J}\right)}{2\left(v+\beta_{v J}\right)\left(v+\gamma_{v J}\right)\left(2 v+\beta_{v J}+\gamma_{v J}+2\right)}, \\
B_{v J}(x)=\frac{v\left[\left(\beta_{v J}-\gamma_{v J}\right)-\left(2 v+\beta_{v J}+\gamma_{v J}\right) x\right]}{2 v+\beta_{v J}+\gamma_{v J}} .
\end{gathered}
$$

\section{Coherent states of the Wei Hua oscillator}

In this section we pay attention to the coherent states of the Wei Hua potential with zero angular momentum. The coherent states can be constructed using the fundamental concepts of supersymmetric quantum mechanics (SUSYQM) [36]. Crucial for this aim is the assumption that Eq. (3) can be transformed to the following canonical SturmLiouville differential equation

$$
-\frac{1}{2} \frac{d^{2} \psi(x)_{v}}{d x^{2}}+\left(\frac{t^{2}(1-Q x)^{2}}{2 x^{2}(1-x)^{2}}-\frac{4 \lambda_{v}+1}{8 x^{2}}\right) \psi(x)_{v}=0,
$$

in which $\psi(x)_{v}$ satisfies the following Liouville transformation 


$$
\Psi(x)_{v}=\psi(x)_{v} \exp \left[-\frac{1}{2} \int \frac{1}{x} d x\right]
$$

Substitution of the wave function (7) into Eq. (48) allows us to obtain

$$
\psi(x)_{v}=x^{\rho_{v}+1 / 2}(1-x)^{1 / 2+\delta} F\left(-v, \beta_{v}, \gamma_{v} ; x\right) .
$$

Let us consider the logarithmic derivative of the ground-state wave function,

$$
W_{0}(x)=\frac{d}{d x} \ln \psi(x)_{0},
$$

which has proven to be useful in classifying most of the exactly solvable Hamiltonians. In supersymmetric quantum mechanics $W_{0}(x)$ is interpreted as a Witten superpotential $[37,38]$. In terms of it we can write

$$
\psi(x)_{0}=\exp \left(\int W_{0}(x) d x\right)
$$

Inserting this function into the Eq. (47) we obtain the Riccati equation which is wellknown in the techniques of SUSYQM [36]

$$
-\frac{1}{2}\left(\frac{d W_{0}(x)}{d x}+W_{0}^{2}(x)\right)+\frac{t^{2}(1-Q x)^{2}}{2 x^{2}(1-x)^{2}}-\frac{4 \lambda v+1}{8 x^{2}}=0 .
$$

Hence, taking into account Eqs. (49) and (50) one may determine

$$
W_{0}(x)=\frac{\frac{1}{2}+\rho_{v}}{x}-\frac{\frac{1}{2}+\delta}{1-x}
$$

Employing the superpotential (53), annihilation and creation operators for the Wei Hua oscillator can be written in the following forms

$$
\hat{A}^{\dagger}=\frac{1}{\sqrt{2}}\left(-\frac{d}{d x}+\frac{\frac{1}{2}+\rho_{v}}{x}-\frac{\frac{1}{2}+\delta}{1-x}\right), \quad \hat{A}=\frac{1}{\sqrt{2}}\left(\frac{d}{d x}+\frac{\frac{1}{2}+\rho_{v}}{x}-\frac{\frac{1}{2}+\delta}{1-x}\right)
$$

Additionally, one may show that the operator $\hat{A}$ annihilates the ground state hence the coherent states $|\alpha\rangle$ of the Wei Hua oscillator are eigenstates of the annihilation operator $\hat{A}$,

$$
\hat{A}|\alpha\rangle=\alpha|\alpha\rangle,|\alpha\rangle=(1-x)^{(1 / 2+\delta)} x^{-\left(1 / 2+\rho_{v}\right)} \exp (\sqrt{2} \alpha x)
$$


It should be stressed that this result is in a excellent agreement with those obtained by Molski [33] who demonstrated that these coherent states minimize the generalized position-momentum uncertainty relation. The normalized coherent states can be presented in a following way

$$
|\alpha\rangle=N_{\alpha} \exp (\sqrt{2} \alpha x) x^{-\left(1 / 2+\rho_{v}\right)}(1-x)^{1 / 2+\delta}
$$

where the normalization constant can be written as

$$
N_{\alpha}=\left[\frac{\sqrt{2} \exp \left(\alpha+\alpha^{*}\right) \Gamma(2+2 \delta) \Gamma\left(-2 \rho_{v}\right)}{\Gamma\left(2+2 \rho_{v}-2 \rho_{v}\right)}\right]^{1 / 2}
$$

The matrix elements in the basis set of the coherent states considered can be analytically calculated using the integral representation of the hypergeometric function [35]

$$
F(a, b, y)=\frac{\Gamma(b)}{\Gamma(a) \Gamma(b-a)} \int_{0}^{1} u^{a-1}(1-u)^{b-a-1} \exp (y u) d u
$$

Taking into account the formula (58) one may easily derive the following matrix elements

$$
\begin{gathered}
\left\langle\alpha\left|\frac{1}{x^{2}}\right| \alpha\right\rangle=N_{\alpha}^{2} \frac{\sqrt{2} \Gamma(2+2 \delta) \exp \left(\alpha+\alpha^{*}\right) \Gamma\left(-2-2 \rho_{v}\right)}{\Gamma\left(-2 \rho_{v}+2 \delta\right)} \\
\left\langle\alpha\left|\frac{1}{(1-x)^{2}}\right| \alpha\right\rangle=N_{\alpha}^{2} \frac{\Gamma(2 \delta) \Gamma\left(-2 \rho_{v}\right)}{\Gamma\left(-2 \rho_{v}+2 \delta\right)}
\end{gathered}
$$

\section{Concluding remarks}

In this paper the ladder operators for the four-parameter Wei Hua potential have been constructed by a simple algebraic procedure developed by Dong et al. [17]. These operators have been obtained directly from the analytical eigenfunctions of the Schrödinger equation for the Wei Hua anharmonic oscillator. Also, in this study the coherent states of the Wei Hua oscillator have been derived using the supersymmetric quantum mechanics. In summary, because this potential may fit to the experimental Rydberg-Klein-Rees curve more closely than other potentials, the constructed ladder operators can be used to compute matrix elements in theoretical spectroscopy. We hope that the raising and lowering operators obtained will inspire other researchers to obtain Barut-Girardello and Perelomov coherent states as well as the Lie dynamical symmetry groups. 
Open Access This article is distributed under the terms of the Creative Commons Attribution License which permits any use, distribution, and reproduction in any medium, provided the original author(s) and the source are credited.

\section{References}

1. I.L. Cooper, J. Phys. A: Math. Gen. 25, 1671 (1992)

2. M.M. Nieto, L.M. Simmons, Phys. Rev. Lett. 41, 207 (1978)

3. S. Kais, R.D. Levine, Phys. Rev. A 41, 1671 (1990)

4. G.C. Gerry, Phys. Rev. A 31, 2721 (1985)

5. G.C. Gerry, Phys. Rev. A 33, 220 (1986)

6. C.V. Sukumar, J. Phys. A L57, 18 (1985)

7. M. de Crombrugghe, V. Rittenberg, Ann. Phys. 99, 151 (1983)

8. V.A. Kostelecky, M.N. Nieto, D. Truax, Phys. Rev. 32, 2627 (1985)

9. LZh Gendenshtein, Eksp. Teor. Fiz. 38, 299 (1983)

10. S.H. Dong, Z. Phys. Chem. 216, 103 (2002)

11. S.H. Dong, Z.Q. Ma, Am. J. Phys. 70, 520 (2002)

12. S.H. Dong, Appl. Math. Lett. 16, 199 (2003)

13. S. Dong, S.H. Dong, Czech. J. Phys. 52, 753 (2002)

14. R.P. Martinez-Y-Romero, H.N. Nunez-Yepez, A.L. Salas-Brito, Int. J. Quantum Chem. 107, 1608 (2007)

15. J. Zuniga, A. Requena, M. Alacid, A. Bastida, Int. J. Quantum Chem. 57, 43 (1996)

16. I.L. Cooper, J. Phys. A: Math. Gen. 25, 1671 (1992)

17. S.H. Dong, R. Lemus, A. Franc, Int. J. Quantum Chem. 83, 433 (2001)

18. S.H. Dong, R. Lemus, Int. J. Quantum Chem. 86, 265 (2002)

19. N.M. Avram, G.E. Drăgănescu, Int. J. Quantum Chem. 64, 655 (1997)

20. S.H. Dong, G.H. Sun, M. Lozada-Cassou, Phys. Lett. 340, 94 (2005)

21. M.M. Nieto, L.M. Simmons, Phys. Rev. Lett. 41, 207 (1978)

22. S. Kais, R.D. Levine, Phys. Rev. A 41, 1671 (1990)

23. M.G. Benedict, B. Molnar, Phys. Rev. A 60, R1737 (1999)

24. A.M. Perelomov, Generalized Coherent States and Their Applications (Springer, Berlin, 1986)

25. S.H. Dong, Can. J. Phys. 80, 129 (2002)

26. M.M. Nieto, L.M. Simmons, Phys. Rev. A 19, 10 (1979)

27. J.R. Klauder, J. Phys. A 29, L293 (1996)

28. J.P. Gazeau, J.R.J. Klauder, Phys. A: Math. Gen. 32, 123 (1999)

29. A.O. Borut, L. Giarardello, Commun. Math. Gen. 21, 41 (1971)

30. M.G. Crawford, A. Phys. Rev. A 62, 012104 (2000)

31. J.-P. Antoine, J.-P. Gazeau, J.R. Klauder, K.A. Penson, J. Math. Phys. 42, 2349 (2001)

32. B. Roy, P. Roy, Phys. Lett. A 296, 187 (2002)

33. M. Molski, J. Phys A: Math. Theor. 42, 165301 (2009)

34. W. Hua, Phys. Rev. A 42, 2524 (1990)

35. I.S. Gradshteyn, I.M. Ryzhik, Tables of Integrals Series and Products, 5th edn. (Academic Press, San Diego, 1994)

36. F. Cooper, A. Khare, U. Sukhatme, Phys. Rep. 251, 267-385 (1995)

37. E. Witten, Nucl. Phys. B 188, 513 (1981)

38. J.J. Peña, M.A. Romero-Romo, J. Morales, J.L. López-Bonilla, Int. J. Quantum Chem. 105, 731 (2005) 